\title{
COMBINATION OF MILK FAT GLOBULE MEMBRANES AND PROBIOTICS TO POTENTIATE NEONATAL IMMUNE MATURATION AND EARLY LIFE PROTECTION
}

\author{
L. Favre ${ }^{1}$, N. Bosco ${ }^{1}$, I. Segura Roggero ${ }^{1}$, B. Corthésy ${ }^{2}$, J. Benyacoub $^{1}$ \\ ${ }^{1}$ Nutrition \& Health, Immunology Group, Nestlé Research Center, ${ }^{2}$ Division of Immunology and Allergy, \\ University State Hospital, Lausanne, Switzerland
}

Background and aims: Education of the neonatal immune system is strongly dependent on environmental stimuli. Milk fat globule membranes (MFGMs) are major component of breast milk and contain molecules with potential immune effects. Probiotics are bacteria with described immune benefits. The aim of the present work was to investigate whether combination of MFGMs and probiotics could be used to promote immune maturation and protection early in life.

Methods: In vitro: Mesenteric lymph node B and T cells proliferation assays (anti-CD3 and anti-CD40 stimulations respectively) were used to assess potentiation of cell immune properties of the combination MFGM-probiotics. In vivo : Freshly weaned mice were daily supplemented for 4 weeks with MFGMs and/or Bifidobacterium lactis probiotic strain CNCM I-3446 or placebo. Number of mucosal IgA secreting cells was analyzed by ELISPOT at the end of the supplementation period and 12 weeks later.

Results: In vitro: Highest mucosal B and T cells proliferative responsiveness was obtained with MFGMs and probiotics, exhibiting stronger effect than MFGM alone or probiotics alone. In vivo : Supplementation with the combination of MFGM and B.Lactis significantly increased the number of mucosal IgA-secreting cells after 28 days of feeding with a greater impact of the combination compared to individual ingredients. Similar effects were observed 12 weeks after the end of the supplementation.

Conclusions: Combination of MFGMs and probiotics demonstrated higher immune modulatory properties than individual ingredients. Early life supplementation with such combination could represent a powerful solution to promote immune maturation and improve protection against early life infections. 